\title{
Agomelatine versus fluoxetine in glycemic control and treating depressive and anxiety symptoms in type 2 diabetes mellitus subjects: a single-blind randomized controlled trial
}

This article was published in the following Dove Press journal:

Neuropsychiatric Disease and Treatment

\author{
Tingting Che ${ }^{1}$ \\ Xiaochun Teng' \\ Qun Huang' \\ Yanfei $\mathrm{Mu}^{2}$ \\ Xianjun Tang ${ }^{2}$ \\ Xiaosong $\mathrm{Mu}^{2}$ \\ Youneng $\mathrm{Wei}^{1}$ \\ 'Department of Endocrinology, \\ Chongqing Public Health Medical \\ Center, Chongqing, China; ${ }^{2}$ Chongqing \\ Cancer Institute, Hospital and Cancer \\ Center, Chongqing, China
}

\begin{abstract}
Background: Depressive and anxiety symptoms could seriously affect the quality of life of type 2 diabetes mellitus (T2DM) subjects. Currently, little is known about the efficacy and acceptability of agomelatine versus fluoxetine in treating these symptoms in T2DM subjects. Therefore, this study was performed to find out which one was better in treating these symptoms in T2DM subjects.
\end{abstract}

Materials and methods: T2DM subjects with depressive and anxiety symptoms were randomly assigned to receive either fluoxetine (30-40 mg/day) or agomelatine (25-50 mg/day). The treatment was continued for 12 weeks. The data of the Hamilton Depression Rating Scale (HDRS) and Hamilton Anxiety Rating Scale (HARS) were collected (at baseline and also at weeks 4, 8 and 12) to assess the depressive and anxiety symptoms, respectively. The metabolic parameters, including body mass index (BMI), fasting plasma glucose (FPG) and hemoglobin $\mathrm{A} 1 \mathrm{c}(\mathrm{HbA1c})$, were assessed at baseline and after 12 weeks of treatment. The treatment-related adverse events during the scheduled treatment period were recorded to compare the acceptability of these two drugs.

Results: After 12 weeks of treatment, the average HDRS and HARS scores were significantly decreased in both groups. The average HDRS scores were not significantly different between the two groups, although the agomelatine group had a lower average HDRS score. The response and remission rates were similar between the two groups, and these two drugs had no significant effects on BMI and FPG. However, compared with the fluoxetine group, the agomelatine group had the significantly lower average HARS score $(p=0.0017)$ and lower average HbA1c level $(p<0.00001)$. Moreover, the incidence of adverse events was significantly lower in the agomelatine group than in the fluoxetine group $(p=0.032)$.

Conclusion: Both fluoxetine and agomelatine could effectively reduce depressive and anxiety symptoms in T2DM subjects, but agomelatine might be more effective and acceptable. Future studies with more subjects are needed to support and validate our conclusion.

Keywords: fluoxetine, agomelatine, diabetes mellitus, depression

\section{Introduction}

Diabetes mellitus (DM) is a common chronic disease with most notably abnormal glucose metabolism. ${ }^{1}$ It affects more than 300 million people worldwide, and about $9 \%$ of the United States population. ${ }^{2}$ Currently, type 2 DM (T2DM) is the most common type of DM. T2DM is highly comorbid with depression, which is a debilitating mental disorder with unclear pathogenesis. ${ }^{3-5}$ Previous studies have shown that about 
$15 \%$ of T2DM subjects were comorbid with depression, ${ }^{6}$ and the rate of life-long prevalence of depression in DM patients was more than $20 \%{ }^{7}$ Meanwhile, the available data show that anxiety frequently coexists with depression. ${ }^{8}$ Researchers found that, compared with T2DM subjects without depressive and anxiety symptoms, T2DM subjects with these symptoms were at greater risk of complications over 5 years. ${ }^{9}$ These two symptoms are commonly associated with a series of adverse diabetes-related outcomes, such as poor glycemic control and problems in self-management. Therefore, it is important to effectively treat the depressive and anxiety symptoms in T2DM subjects.

Up to now, many treatment modalities have been developed to treat the depressive and anxiety symptoms, but pharmacotherapy is still the preferred treatment method. ${ }^{10}$ However, clinicians should take good care in choosing the antidepressants, because some antidepressants might be inappropriate for treating the depressive and anxiety symptoms in DM subjects, such as monoamine oxidase inhibitors and tricyclic antidepressants. ${ }^{11,12}$ The former has the possibility to cause weight gain, ${ }^{11}$ and the latter might result in hyperglycemia, carbohydrate intake increase, and memory impairment. ${ }^{12}$ Therefore, when using antidepressants to treat the depressive and anxiety symptoms in DM subjects, clinicians should evaluate the effects of antidepressants on glycemic control. ${ }^{11,12}$

A previous study showed that the selective serotonin reuptake inhibitors (SSRIs) were well tolerated and seemed to have some advantages in treating depressive and anxiety symptoms in DM patients. ${ }^{13}$ The available evidence seems to support the use of fluoxetine in treating the depressive symptoms in T2DM subjects. ${ }^{14-16}$ Moreover, Potter van Loon et al reported that fluoxetine could improve peripheral and hepatic insulin action in obese insulin-resistant patients. ${ }^{17}$ In addition, agomelatine, as a new antidepressant, was reported to offer some advantages over sertraline in treating depressive and anxiety symptoms in T2DM subjects, as well as improving their health-related behaviors. ${ }^{18}$ However, few studies have attempted to investigate which one, fluoxetine or agomelatine, could produce better efficacy for T2DM subjects with depressive and anxiety symptoms. Therefore, this study was conducted to compare the efficacy and acceptability of agomelatine and fluoxetine in treating the depressive and anxiety symptoms in T2DM subjects, as well as their efficacy on glycemic control.

\section{Materials and methods} Subject selection

This study was approved by the Ethical Committee of Chongqing Public Health Medical Center, and the methods were carried out according to the approved guidelines and regulations. This study was conducted between January 2015 and June 2017 in Chongqing Public Health Medical Center. T2DM subjects meeting the following criteria were recruited by two experienced clinicians: 1) a value of hemoglobin A1c $(\mathrm{HbA} 1 \mathrm{c})>7.0 \%$; 2) a 17-item Hamilton Depression Rating Scale (HDRS-17) score $\geqq 17$ and Hamilton Anxiety Rating Scale (HARS) score $>7 ;^{19,20} 3$ ) 18-80 years of age; 4 ) in order to exclude T2DM subjects with cognitive impairment, the Mini Mental State Examination score of each subject should be more than 24; and 5) without active suicidal ideation, a history of any other psychiatric disorders, and serious mental illness or physical health problems. Meanwhile, T2DM subjects were excluded if they previously or currently received psychoactive medications. All T2DM subjects provided the written informed consent before randomization.

\section{Study design}

This study was a two-arm, single-blind randomized controlled trial. The included T2DM subjects were randomly assigned into two groups using a separate computer-generated random number sequence. T2DM subjects in the fluoxetine group received fluoxetine $30-40 \mathrm{mg} / \mathrm{day}$, and T2DM subjects in the agomelatine group received agomelatine $25-50 \mathrm{mg} /$ day. The treatment was continued for 12 weeks. The HDRS score and HARS score were assessed at baseline and also at weeks 4 , 8, and 12. The HbAlc, body mass index (BMI) and fasting plasma glucose (FPG) were assessed at baseline and after 12 weeks of treatment. The BMI was calculated as body weight $(\mathrm{kg})$ divided by the square of the height $\left(\mathrm{m}^{2}\right)\left(\mathrm{kg} / \mathrm{m}^{2}\right)$. Although T2DM subjects were not blind to the drugs being given (fluoxetine/agomelatine), the raters were blind to the drug regimen of T2DM subjects to reduce the potential bias. At the end of the trial, T2DM subjects that did not respond to these two drugs would continually receive psychological treatment or medications.

\section{Main outcome measures}

The HDRS and HARS scores were viewed as the primary outcomes, which were used to assess the efficacy of these two drugs in treating the depressive and anxiety symptoms, respectively. The response and remission rates were viewed as the secondary outcomes. The response was defined as a $>50 \%$ reduction from the baseline HDRS-17 score, and the remission was defined as an absolute HDRS-17 score $\leq 7$ at the end of the trial. ${ }^{21}$ The HbA1c, BMI, and FPG were also viewed as the secondary outcomes, which were used to assess the efficacy of agomelatine and fluoxetine on glycemic control. Meanwhile, the treatment-related adverse events during 
the scheduled treatment period were recorded to assess the acceptability of agomelatine and fluoxetine.

\section{Statistical analyses}

Statistical analysis was conducted with SPSS version 19.0 for Windows (IBM Corporation, Armonk, NY, USA). The Chisquare and Student's $t$-test were used to investigate the group differences on demographic data. The repeated measures analysis of variance (ANOVA) was conducted to investigate the group differences on HDRS and HARS scores at four time points. The analysis of covariance (ANCOVA) was conducted to investigate the group differences on $\mathrm{HbA} 1 \mathrm{c}$, BMI, and FPG at weeks 12, with their scores at baseline as a covariate. ${ }^{22,23}$ All statistical tests were two-sided and a $p$-value less than 0.05 was considered statistically significant.

\section{Results}

\section{Baseline characteristics}

A total of 95 T2DM subjects met the above-mentioned inclusion criteria. After excluding eight T2DM subjects refusing randomization and three T2DM subjects refusing any treatments, the remaining 84 T2DM subjects were included in this study and randomly assigned to receive fluoxetine $(n=40)$ or agomelatine $(\mathrm{n}=44)$. During the scheduled treatment period, four T2DM subjects in the fluoxetine group failed to complete the trial: one subject withdrew from the study because of economic reasons; one subject was excluded because of receiving psychological therapy at week 8 ; and we lost contact with two subjects after 8 weeks of treatment. Meanwhile, three T2DM subjects in the agomelatine group failed to complete the trial: two subjects withdrew from the study because of economic reasons; one subject refused to continue to receive treatment after 10 weeks of treatment. The differences of demographic and clinical variables between the two groups, such as average age, sex ratio, education level, BMI, and duration of T2DM, were not statistically significant. The detailed information of the included T2DM subjects is described in Table 1.

\section{Primary outcomes}

As shown in Figure 1, the mean changes in HDRS scores from baseline to the end of the trial were -12.53 and -13.87 in the fluoxetine group and agomelatine group, respectively. The results of repeated measures ANOVA showed a significant effect of time $(p<0.00001)$, which indicated that both fluoxetine and agomelatine could significantly reduce the HDRS scores in T2DM subjects. Meanwhile, the results of repeated measures ANOVA showed no significant treatment group main effect ( $p=0.095)$, which indicated that the reductions of HDRS scores were not significantly different between
Table I Demographic profile of the recruited subjects

\begin{tabular}{llll}
\hline Variables & Fluoxetine & Agomelatine & p-value \\
\hline $\mathrm{n}$ & 40 & 44 & - \\
Age (years) & $52.65 \pm 10.77$ & $5 \mathrm{I} .66 \pm 10.30$ & 0.67 \\
Female/Male, $\mathrm{n}$ & $25 / 15$ & $23 / 2 \mathrm{I}$ & 0.34 \\
Education (years) & $9.18 \pm 3.12$ & $9.36 \pm 3.10$ & 0.78 \\
$\mathrm{BMI}\left(\mathrm{kg} / \mathrm{m}^{2}\right)$ & $25.72 \pm 3.27$ & $25.37 \pm 3.68$ & 0.64 \\
$\mathrm{FPG} \mathrm{(mmoL/L)}$ & $8.10 \pm 0.77$ & $8.13 \pm 0.79$ & 0.89 \\
HbAlc (\%) & $7.99 \pm 0.44$ & $7.90 \pm 0.40$ & 0.35 \\
Duration of diabetes (years) & $8.02 \pm 4.38$ & $8.30 \pm 4.52$ & 0.77 \\
Smoking (Y/N), n & $8 / 32$ & $10 / 34$ & 0.76 \\
Dose (mg/day) & $34.50 \pm 5.04$ & $39.32 \pm 8.18$ & - \\
HDRS scores & $23.88 \pm 2.93$ & $23.66 \pm 2.96$ & 0.73 \\
HARS scores & $15.80 \pm 2.77$ & $15.57 \pm 3.62$ & 0.74 \\
\hline
\end{tabular}

Note: Data shown as mean \pm standard deviation unless indicated otherwise.

Abbreviations: BMI, body mass index; FPG, fasting plasma glucose; HbAlc, hemoglobin AIc; HDRS, Hamilton Depression Rating Scale; HARS, Hamilton Anxiety Rating Scale; Y, yes; N, no.

the two groups. These results showed that agomelatine $25-50 \mathrm{mg} /$ day was equivalent to fluoxetine $30-40 \mathrm{mg} /$ day in treating depressive symptoms in T2DM subjects, although the subjects receiving agomelatine had a lower average HDRS score after 8 and 12 weeks of treatment.

As shown in Figure 2, the mean changes in HARS scores from baseline to the end of the trial were -5.60 and -7.11 in the fluoxetine group and agomelatine group, respectively. The results of repeated measures ANOVA showed a significant effect of time $(p<0.00001)$, which indicated that both fluoxetine and agomelatine could significantly reduce the HARS scores in T2DM subjects. Meanwhile, the results of repeated measures ANOVA showed a significant treatment group main effect ( $p=0.005$ ), which indicated that the reductions of HARS scores were significantly different between the two groups. These results showed that, compared with fluoxetine, agomelatine was significantly more effective in treating the anxiety symptoms in T2DM subjects.

\section{Secondary outcomes}

After 12 weeks of treatment, there were 17 T2DM subjects meeting the response criteria and nine T2DM subjects meeting the remission criteria in the fluoxetine group. Meanwhile, there were $22 \mathrm{~T} 2 \mathrm{DM}$ subjects meeting the response criteria and $11 \mathrm{~T} 2 \mathrm{DM}$ subjects meeting the remission criteria in the agomelatine group. Both the response rates and remission rates were similar between the two groups (response rate, $p=0.491$; remission rate, $p=0.788$ ), although the agomelatine group had the higher response rate and remission rate.

As shown in Figure 3, at baseline, the BMI was similar between the two groups ( $p=0.645)$. After 12 weeks of treatment, the BMI was not significantly changed in both the fluoxetine group ( $p=0.761)$ and agomelatine group ( $p=0.576)$. Meanwhile, the results of ANCOVA showed that the BMI 


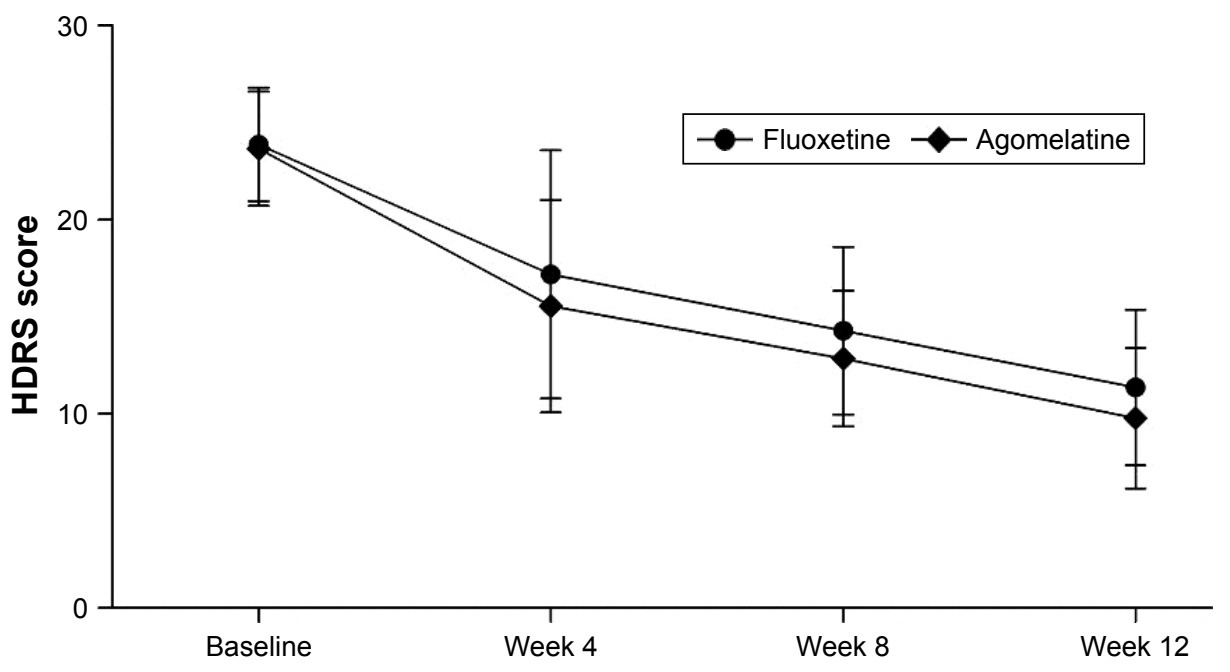

Figure I Average HDRS scores at four time points.

Note: Error bars represent standard deviation.

Abbreviation: HDRS, Hamilton Depression Rating Scale.

was still similar between the two groups after 12 weeks of treatment $(p=0.105)$. Similar results were observed on FPG. These results indicated that these two drugs had no significant effects on the weight and FPG of T2DM subjects.

At baseline, the HbA1c level was similar between the two groups $(p=0.352)$. After 12 weeks of treatment, the HbA1c level was significantly decreased in both the fluoxetine group ( $p=0.005)$ and agomelatine group $(p<0.00001)$ compared with their initial scores. However, as shown in Figure 3, the results of ANCOVA showed that the average HbA1c level after 12 weeks of treatment was significantly lower in the agomelatine group than in the fluoxetine group $(p<0.00001)$. These results showed that, compared with fluoxetine, agomelatine was significantly more effective in reducing the $\mathrm{HbA} 1 \mathrm{c}$ level of T2DM subjects.

\section{Adverse events}

During the scheduled treatment period, two T2DM subjects in the fluoxetine group developed acute suicidal intent at week 10; however, there were no suicides. Meanwhile, no T2DM subject in the agomelatine group developed acute suicidal intent. The treatment-related adverse events in the two groups during the scheduled treatment period were described as follows: fluoxetine/agomelatine (n, \%): hyperhidrosis $0(0 \%) / 2(4.6 \%)$, nausea $4(10 \%) / 2(4.6 \%)$, headache $2(5 \%) / 1(2.3 \%)$, vomiting $1(2.5 \%) / 1(2.3 \%)$, sexual side

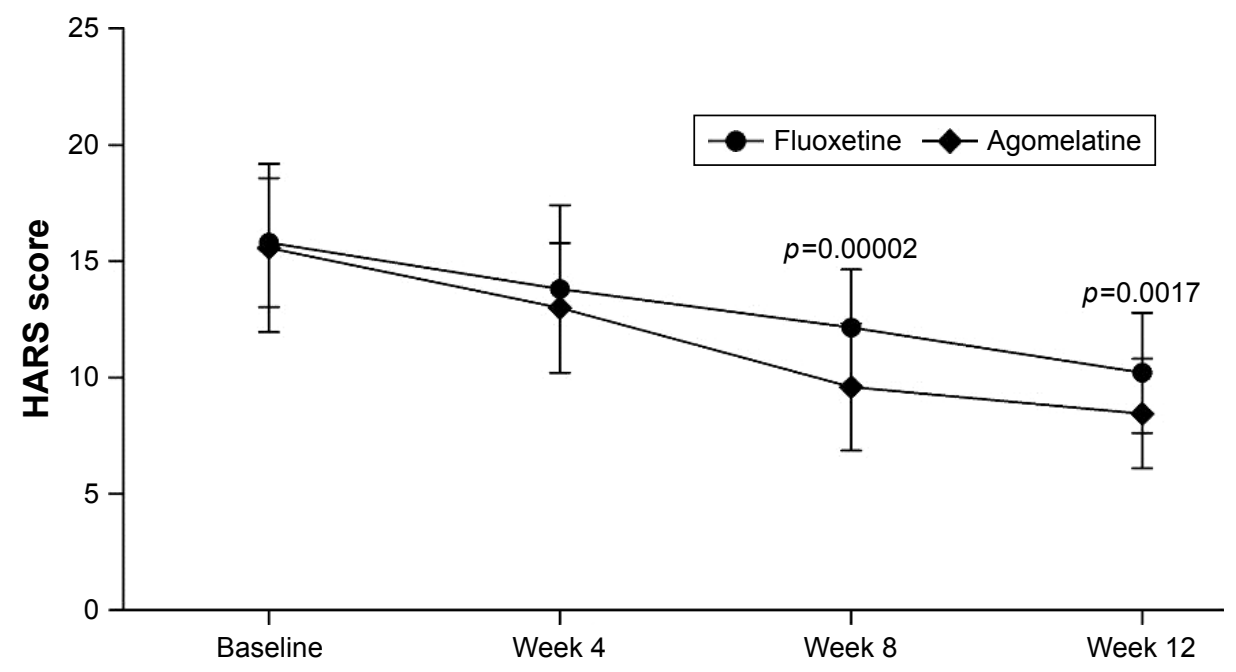

Figure 2 Average HARS scores at four time points.

Note: Error bars represent standard deviation.

Abbreviation: HARS, Hamilton Anxiety Rating Scale. 

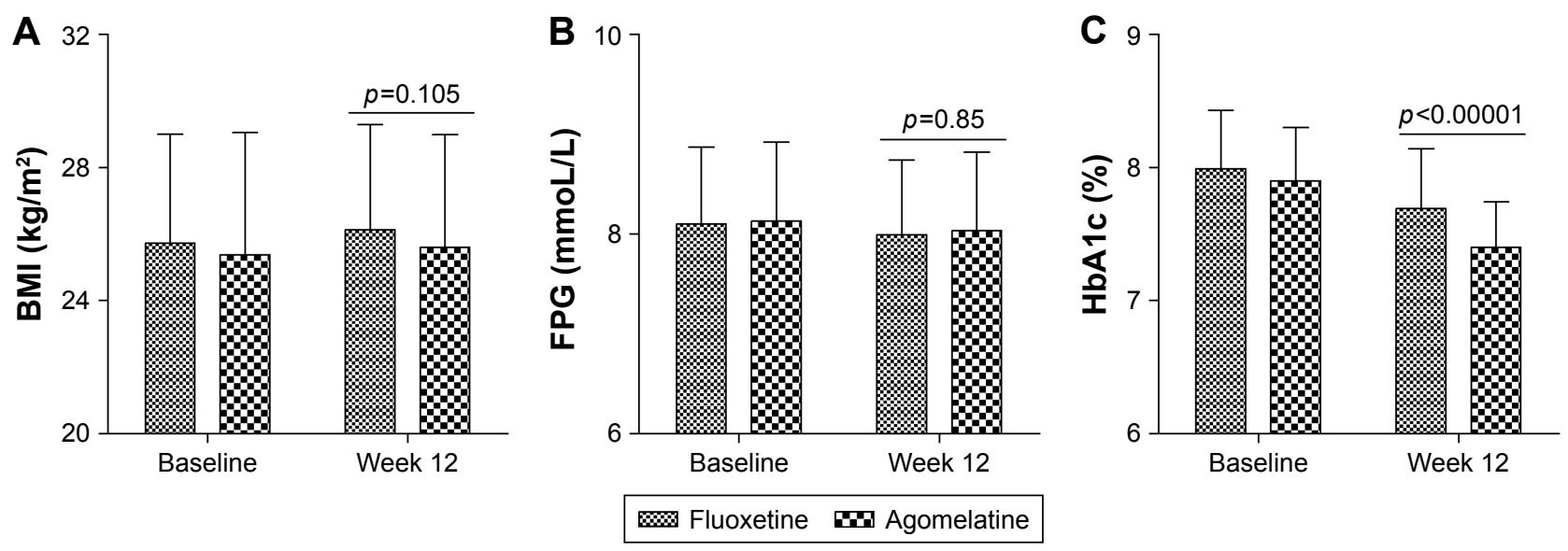

Figure 3 Average BMI (A), FPG (B), and $\mathrm{HbAlc}(\mathbf{C})$ at baseline and at week 12 .

Note: Error bars represent standard deviation.

Abbreviations: BMI, body mass index; FPG, fasting plasma glucose; HbAlc, hemoglobin Alc.

effects $2(5 \%) / 0(0 \%)$, acute suicidal intent $2(5 \%) / 0(0 \%)$, dizziness $2(5 \%) / 1(2.3 \%)$, anorexia $2(5 \%) / 0(0 \%)$, diarrhea $2(5 \%) / 2(4.6 \%)$, and insomnia $3(7.5 \%) / 1(2.3 \%)$. The liver function was monitored at baseline and also at weeks 4,8 , and 12. The incidence of treatment-related adverse events was significantly different between the two groups $(p=0.032)$.

\section{Discussion}

In this study, we found that both fluoxetine and agomelatine could effectively treat depressive and anxiety symptoms in T2DM subjects. However, compared with fluoxetine, agomelatine could yield a non-significantly lower average HDRS score and a significantly lower average HARS score after 12 weeks of treatment. The response rates and remission rates were similar between the two groups. The BMI and FPG were not significantly changed after 12 weeks of treatment, which indicated that the two drugs had no significant effects on these variables. In addition, the average $\mathrm{HbA} 1 \mathrm{c}$ level in the agomelatine group was significantly lower when compared with the fluoxetine group $(p<0.00001)$. Meanwhile, we found that agomelatine might be more acceptable in treating T2DM subjects. Therefore, these results suggested that both fluoxetine and agomelatine could effectively treat depressive and anxiety symptoms in T2DM subjects, but agomelatine might be more effective and acceptable.

The anorectic effect of fluoxetine is independent from its antidepressant effect, which makes it very often used in T2DM patients with depressive and anxiety symptoms. ${ }^{15,16}$ In addition to leading to the decrease of HDRS score and loss of weight, the positive effect of fluoxetine on glycemic control has also been reported by previous studies. ${ }^{24,25}$ Consistent with these findings, our study found that fluoxetine-treated
T2DM subjects had a good glycemic control with a significant reduction of $\mathrm{HbA} 1 \mathrm{c}$ levels. Meanwhile, our results showed that agomelatine could produce a trend toward better glycemic control than fluoxetine after 12 weeks of treatment. The reduction of $\mathrm{HbA} 1 \mathrm{c}$ level in the agomelatine group was significantly more when compared with the fluoxetine group. Therefore, agomelatine might be superior to fluoxetine in terms of its effect on HbAlc levels. However, due to the relatively small sample size, future large-scale studies are needed to support and validate our conclusion.

A recent study reported that agomelatine, as a melatonergic antidepressant, could significantly decrease the final HbA1c levels of depressed patients with T2DM after a 4-month treatment. ${ }^{18}$ Compared with SSRIs, agomelatine would not produce discontinuation syndrome or sexual side effects. Moreover, it might also have positive effects on sleep. Previous studies also showed that agomelatine was at least as effective as SSRIs, such as escitalopram and fluoxetine, in patients with depression. ${ }^{26,27}$ A large meta-analysis including 7,460 participants found that agomelatine was as effective as standard antidepressants. ${ }^{28}$ Aydin and colleagues found that both agomelatine and fluoxetine were efficacious in the treatment of depression, and agomelatine could be an appropriate choice for patients with depression. ${ }^{29}$ Our study found that agomelatine was more effective and acceptable than fluoxetine. Meanwhile, body weight gain was not observed in the agomelatine group, which might be helpful in maintaining better glycemic control. ${ }^{30}$ Therefore, agomelatine might be a promising agent for treating the depressive and anxiety symptoms in T2DM subjects.

Limitations of this study should be addressed here. Firstly, the sample size was relatively small, which might 
have limited available power and ability to detect the subtler changes. Secondly, the treatment was only continued for 12 weeks; thus, future studies are needed to measure these outcomes over longer periods. Thirdly, we only investigated the efficacy and acceptability of these two drugs here, additional studies that compare agomelatine with other treatment methods could help to find out which treatment methods are likely to benefit particular subjects. Fourthly, the impact of changes in diabetes medications (insulin and oral medications) on the health outcomes during the scheduled treatment period was not assessed in this study. Fifthly, the compliance was not assessed in this study. Finally, T2DM subjects were not blind to the drugs being given (fluoxetine/agomelatine), which might cause potential bias in our conclusion. Therefore, future studies (double-blind design) are needed to verify and support our conclusion.

In conclusion, both fluoxetine and agomelatine were effective in treating the depressive and anxiety symptoms in T2DM subjects. But, compared with fluoxetine, agomelatine might be more effective and acceptable in treating T2DM subjects with depressive and anxiety symptoms, and should be further explored.

\section{Disclosure}

The authors report no conflicts of interest in this work.

\section{References}

1. Nathan DM. Long-term complications of diabetes mellitus. $N$ Engl J Med. 1993;328(23):1676-1685.

2. Eke PI, Wei L, Thornton-Evans GO, et al. Risk indicators for periodontitis in US adults: NHANES 2009 to 2012. J Periodontol. 2016;87(10): 1174-1185.

3. Chen J, Zheng P, Liu Y, et al. Sex differences in gut microbiota in patients with major depressive disorder. Neuropsychiatr Dis Treat. 2018; 14:647-655.

4. Chen JJ, Zhou CJ, Zheng P, et al. Differential urinary metabolites related with the severity of major depressive disorder. Behav Brain Res. 2017;332:280-287.

5. Chen JJ, Zeng BH, Li WW, et al. Effects of gut microbiota on the microRNA and mRNA expression in the hippocampus of mice. Behav Brain Res. 2017;322(Pt A):34-41.

6. Golden SH, Lazo M, Carnethon M, et al. Examining a bidirectional association between depressive symptoms and diabetes. JAMA. 2008;299(23):2751-2759.

7. Eiber R, Berlin I, Grimaldi A, Bisserbe JC. [Insulin-dependent diabetes and psychiatric pathology: general clinical and epidemiologic review.] Encephale. 1997;23(1):351-357. [In French].

8. Agius A, Xuereb RB, Carrick-Sen D, Sultana R, Rankin J. The coexistence of depression, anxiety and post-traumatic stress symptoms in the perinatal period: a systematic review. Midwifery. 2016;36:70-79.

9. Roglic G, Unwin N. Mortality attributable to diabetes: estimates for the year 2010. Diabetes Res Clin Pract. 2010;87(1):15-19.

10. Wolf NJ, Hopko DR. Psychosocial and pharmacological interventions for depressed adults in primary care: a critical review. Clin Psychol Rev. 2008;28(1):131-161.
11. Rabkin J, Qutkin F, Harrison W, Tricamo E, McGrath P. Adverse reactions to monoamine oxidase inhibitors. I. A comparative study. J Clin Psychopharmacol. 1984;4(5):270-278.

12. Hindmarch I. The psychopharmacological approach: effects of psychotropic drugs on car handling. Int Clin Psychopharmacol. 1988;3(Suppl 1): 73-79.

13. Gehlawat P, Gupta R, Rajput R, Gahlan D, Gehlawat VK. Diabetes with comorbid depression: role of SSRI in better glycemic control. Asian J Psychiatr. 2013;6(5):364-368.

14. Goodnick PJ. Use of antidepressants in treatment of comorbid diabetes mellitus and depression as well as in diabetic neuropathy. Ann Clin Psychiatry. 2001;13(1):31-41.

15. Lustman PJ, Freedland KE, Griffith LS, Clouse RE. Fluoxetine for depression in diabetes: a randomized, double-blind, placebo-controlled trial. Diabetes Care. 2000;23(5):618-623.

16. Gülseren L, Gülseren S, Hekimsoy Z, Mete L. Comparison of fluoxetine and paroxetine in type II diabetes mellitus patients. Arch Med Res. 2005;36(2):159-165.

17. Potter van Loon BJ, Radder JK, Frolich M, Krans HM, Zwinderman AH, Meinders AE. Fluoxetine increases insulin action in obese type II (noninsulin dependent) diabetic patients. Int J Obes Relat Metab Disord. 1992;16(Suppl 4):S55-S61.

18. Karaiskos D, Tzavellas E, Ilias I, Liappas I, Paparrigopoulos T. Agomelatine and sertraline for the treatment of depression in type 2 diabetes mellitus. Int J Clin Pract. 2013;67(3):257-260.

19. Chen JW, Xie SQ. Agomelatine versus paroxetine in treating depressive and anxiety symptoms in patients with chronic kidney disease. Neuropsychiatr Dis Treat. 2018;14:547-552.

20. Kang R, He Y, Yan Y, et al. Comparison of paroxetine and agomelatine in depressed type 2 diabetes mellitus patients: a double-blind, randomized, clinical trial. Neuropsychiatr Dis Treat. 2015;11:1307-1311.

21. Kent JM, Daly E, Kezic I, et al. Efficacy and safety of an adjunctive mGlu2 receptor positive allosteric modulator to a SSRI/SNRI in anxious depression. Progr Neuropsychopharmacol Biol Psychiatry. 2016; 67:66-73.

22. Chilton R, Tikkanen I, Cannon CP, et al. Effects of empagliflozin on blood pressure and markers of arterial stiffness and vascular resistance in patients with type 2 diabetes. Diabetes Obes Metab. 2015;17(12): 1180-1193.

23. Jamieson J. Analysis of covariance (ANCOVA) with difference scores. Int J Psychophysiol. 2004;52(3):277-283.

24. Daubresse JC, Kolanowski J, Krzentowski G, Kutnowski M, Scheen A, Van Gaal L. Usefulness of fluoxetine in obese non-insulin-dependent diabetics: a multicenter study. Obes Res. 1996;4(4):391-396.

25. Connolly VM, Gallagher A, Kesson CM. A study of fluoxetine in obese elderly patients with type 2 diabetes. Diabet Med. 1995;12(5): 416-418.

26. Kasper S, Hajak G, Wulff K, et al. Efficacy of the novel antidepressant agomelatine on the circadian rest-activity cycle and depressive and anxiety symptoms in patients with major depressive disorder: a randomized, double-blind comparison with sertraline. J Clin Psychiatry. 2010;71(2):109-120.

27. Singh SP, Sing V, Kar N. Effcacy of agomelatine in major depressive disorder: meta-analysis and appraisal. Int $J$ Neuropsychopharmacol. 2012;15(3):417-428

28. Taylor D, Sparshatt A, Varma S, Olofinjana O. Antidepressant efficacy of agomelatine: meta-analysis of published and unpublished studies. BMJ. 2014;348:g1888.

29. Aydın E, Güleç M, Oral E, Daloğlu AG. Agomelatine vs fluoxetine: efficacy and improvement of cognitive functions in patients with MDD. Eur Psychiatry. 2016;33(Suppl):S501-S502.

30. Tamura Y, Tanaka Y, Sato F, et al. Effects of diet and exercise on muscle and liver intracellular lipid contents and insulin sensitivity in type 2 diabetic patients. J Clin Endocrinol Metab. 2005;90(6):3191-3196. 


\section{Publish your work in this journal}

Neuropsychiatric Disease and Treatment is an international, peerreviewed journal of clinical therapeutics and pharmacology focusing on concise rapid reporting of clinical or pre-clinical studies on a range of neuropsychiatric and neurological disorders. This journal is indexed on PubMed Central, the 'PsycINFO' database and CAS, and is the official journal of The International Neuropsychiatric Association (INA). The manuscript management system is completely online and includes a very quick and fair peer-review system, which is all easy to use. Visit http://www.dovepress.com/testimonials.php to read real quotes from published authors.

\footnotetext{
Submit your manuscript here: http://www.dovepress.com/neuropsychiatric-disease-and-treatment-journal
} 